\title{
ESTIMATION OF CORAL RUBBLE QUANTITY USING EDGE DETECTION AND MORPHOLOGICAL- BASED METHODS
}

\section{NURSYAHIRAH HAFIZ ${ }^{1}$, MUHAMMAD SUZURI HITAM ${ }^{2 *}$, ZAINUDDIN BACHOK $^{3}$ AND MOHD SAFUAN CHE DIN ${ }^{4}$}

\author{
${ }^{1,2}$ School of Informatics and Applied Mathematics, Universiti Malaysia Terengganu, \\ 21030 Kuala Nerus, Terengganu, \\ ${ }^{3}$ School of Marine and Environmental Sciences, Universiti Malaysia Terengganu, \\ 21030 Kuala Nerus, Terengganu \\ ${ }^{3,4}$ Institute of Oceanography \& Environment (INOS), Universiti Malaysia Terengganu, \\ 21030 Kuala Nerus, Terengganu
}

\section{*Corresponding author: suzuri@umt.edu.my}

\begin{abstract}
Reef rubble represents the broken components of the coral and reef structure which could be in the form of dead, broken or other fragmented coral. The process to estimate the distribution of reef rubble is currently done manually and thus takes a long time to complete and is laborious. This paper presents an image-processing-based method to estimate the distribution of reef rubbles in a coral reef environment from a still image. The method is basically a series of image processing steps including image complement, image binarization, edge detection, smoothing by Weiner filter and followed by erosion and dilation process. The experimental results showed that the system was able to roughly estimate the distribution of reef rubble.
\end{abstract}

Keywords: Reef rubble, Edge detection, Morphological method and Image processing.

\section{Introduction}

Coral reefs which are known as "Rainforests of the Sea" are underwater ecosystems that have been formed from the last glacial period. Coral reefs are built by some of marine water animals and also by stony corals. Corals have the ability to support and protect the reef because they can secrete hard carbonate exoskeletons. Coral reefs play many important roles such as protecting the coastline from wave actions, facilitating in carbon and nitrogen fixing, providing shelter and habitat to many marine organisms and others (Alcala and Gomez, 1982).

Nowadays, the availability of coral reefs around the globe is reducing mainly due to climate change effect, environmental pollution and man-made activities. The health of coral reefs is greatly affected by the condition of surrounding environment. When the sunlight is blocked from reaching the seafloor, the growth of algae is affected and consequently the growth of coral reefs is also affected. In addition, the increase in sea water temperature will also lead to coral bleaching. This phenomenon among others threatens the health of coral reef and may lead to mortality. When the health condition of the coral reefs is affected, it may lead to broken reef components called coral rubble.

In this paper, reef rubble represents the broken components of the coral reefs which could be in the form of dead corals, broken corals, bleached corals, digitate corals, foliose corals and other fragments of coral reefs. Reef rubble shows how much damaged components are there in the coral reef environment. Reef rubble has different shapes and sizes according to where it came from and how it was formed. Rasser and Riegl (2002) defined reef rubble as fraction parts of reef structure larger than the sand. Reef rubble can be formed by strong 
underwater current, wave turbulence, bioerosions, human activities and uncontrolled fisheries.

The more volume of reef rubble in a coral reef environment, the unhealthier the condition is. This is why the coral reefs around the globe are decreasing. An initiative that can help is by mapping the coral reefs. Marine scientists have been mapping coral reefs to gather important information such as overall structure and morphology. In order to estimate the components in coral reefs, scientists have to use many methods such as multispectral imagery (Bierwirth, Lee and Burne, (1993), Coral Video Transect (Carleton \& Done, 1995) and remote sensing (Benediktsson, Pesaresi and Amason, 2003). Kohler \& Gill (2006) introduced a method to estimate the distribution of coral reefs by using Microsoft Excel software. With their method, the marine scientists are able to manually estimate the coral reefs distribution by marking on the still image at some selected random points. This manual process still takes long hour of manual labour to complete the whole recorded video transects. Therefore, this paper presents a new method to estimate the quantity of reef rubble from still image sample.

\section{Literature Review}

Reef rubble is either dead, broken components or fragments of coral reefs. Woodley et al. (1981) reported that the amount of reef rubble formed depends on many factors such as shapes, sizes and mechanical properties of the structures. In order to estimate the reef rubble components, there are several methods that have been used by marine scientists. Green et al. (1996) reported that most popular method for coral reef survey is by using remote sensing technology due to the fact that vast area of survey coverage can be made with a single hyper spectral images. The resolution of the images will determine the accuracy of the method; a low resolution image will only be able to coarsely differentiate between the seagrass and coral reefs and on the other hand, the high resolution images will be able to discriminate between the different type of coral reef species (Mumby and Harborne, 1999).

Marine scientists can assess the condition of coral reefs through many methods. In the past, the regular method was on site diving survey carried out by trained divers. This method takes huge amount of time and energy due to the vast survey area. Recently, this onsite survey has been replaced by underwater digital video recording, and coral reef mapping survey is carried out in the laboratory, speeding up the survey time and most importantly providing a reliable record keeping for future references (Alcala \& Gomez, 1982). Some researchers prefer to use airborne multispectral cameras (Bierwirth et al., 1993; Carper et al., 1990; Chavez et al., 1991; Mausel et al., 1990), but this method cannot provide all the closed up detail of the coral environment (Soriano et al., 2001). Thus, this is the reason why digital video recording or photographic method has been popularly used today by many researchers (Kohler and Gill, 2006).

Digital video recording involves recording a video transect at approximately fixed depth from the seafloor over a certain line transect. Almost similar to the diving method, the random sampling is made on the still images at selected image frame (Lenth, 2001). The technique first converts the recorded video images into sequences of still images and followed by disbursing a set of random points onto the selected still image. An expert marine operator will map the random points onto the specific type of coral reef at each point. By analysing the whole video images, the mapping of each type of coral reef species is made over a region of interest. Coral Point Count with Excel extensions (CPCe) developed by Kohler and Gill (2006) basically provides these facilities to enable marine researchers to carry out the coral reef mapping survey in more reliable and efficient environment. However, most of the activities conducted using CPCe are done manually, so the process of estimating the distribution of the coral reefs from the video transect takes a long time to complete. 
Those are the conventional approach in coral reef distribution estimation. As time goes by, the marine scientists need a new approach that is able to speed up the current manual process. With the advancement of computing technology, one of the ways is to use image processing to estimate the reef rubble distribution more efficiently. There are many image processing techniques that can be employed in coral reef survey such as image quality enhancement, and coral reef type identification and recognition, to name a few. Soriano et al. (2001) used a feed forward multilayer peceptron neural network to classify five benthic types of coral reefs using a digital video recording media. In general, they found that using textured-based features provided better results compared to using only colour or combination of texture and colour features.

Besides colour and texture features, coral reefs can be recognized using shape based features. Among the straightforward and popularly used shape based technique is by using edge detection (Hussain and Agarwal, 2015). Edge detection works by detecting sharp intensity changes across an image. There are many edge detection methods in the literature that can be used for coral reef recognition, such as Sobel, Robert's cross, Prewitt, Laplacian of Gaussian (LoG) and Canny edge detection.

\section{Materials and Methods Research Methodology}

In this paper, the sample image was first preprocessed by complementing the colour of the image. After the colour complement process of the RGB image, the dull regions of the image would wind up lighter and light territories of the image would end up darker. Image complement process is vital due to the natural colour of sand that is white, so when light territories become darker, sand cannot be clearly observed in the image. However, there are situations where image complement process is not sufficient to remove the sand fully. This leads to the next step, which is image segmentation.
As its name implies, image segmentation process segments a digital image into different regions based on the property of the region. The objective of segmentation process is to segment the object of interest from other background objects. This is done by thresholding the output image. Image thresholding is a technique to segment an image into a foreground and background that can separate unwanted objects by changing the RGB image into binary image. Image thresholding works best in images with elevated amount of difference. Due to the output image's intense colour, the thresholding process is able to separate the rubble from other objects to some extent. The following step in image segmentation is edge detection. Edge detection works by identifying discontinuities in brightness of the object in the image.

The following step is feature extraction process. Before the process of feature extraction can be done successfully, the image needs to be pre-processed to remove image noise. Image noise is an irregular variation of brightness that exists in images. This may be caused by the sensor and circuitry of a camera or the presence of floating solid particles in the underwater environment. In order to remove these noises, noise smoothing is employed. Noise smoothing is a technique whereby it reduces the effects of irregular variation of brightness. In this work, the filter used for noise smoothing was Wiener filter. In image processing, Wiener filter is a filter used to remove additive noises and deblur image simultaneously.

In this work, an object was considered as rubble if it was less than $5 \mathrm{~cm}$ in length. After a few preliminary studies made, by using a morphological-based method which were erosion and dilation, rubble could potentially be detected. Erosion and dilation are basic morphological methods used to remove or add pixels to the boundaries of objects in an image. With the use of structural element that can be set to a proper measurement for detecting rubbles, erosion and dilation can provide promising results. 
To estimate area of the rubble coverage, a basic coding in MATLAB was developed. Since the image only consisted of black and white pixels, the black pixel represented the unwanted and unnecessary objects in the image while the white pixels represented the potential rubble coverage. The rubble coverage could be counted by counting the white pixels over the total pixel of image.

Lastly, a simple interface with a few buttons was created by using GUI feature in

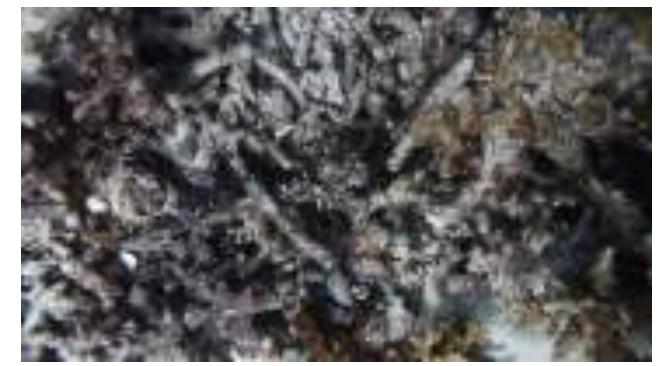

(a)

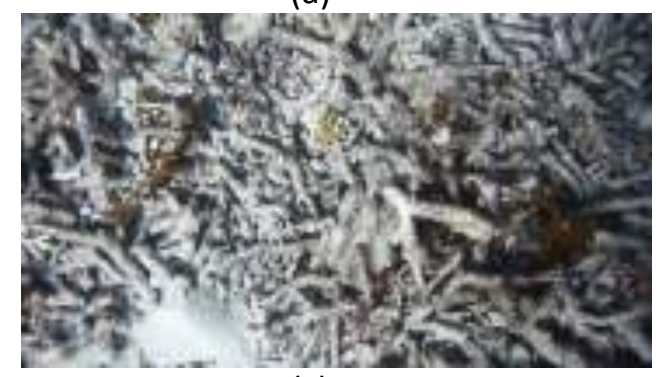

(c)

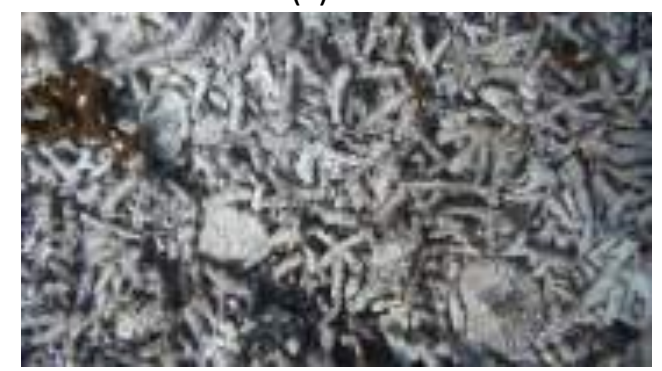

(e)
MATLAB software to ease the users. The interface will automatically show the results for any images selected by user.

\section{Data Collection}

Image dataset used in this work were collected from the Institute of Oceanography and Environment (INOS), Universiti Malaysia of Terengganu (UMT). Figure 1(a), (b), (c), (d), (e) and (f) show a few image samples of rubble in coral reef environment.

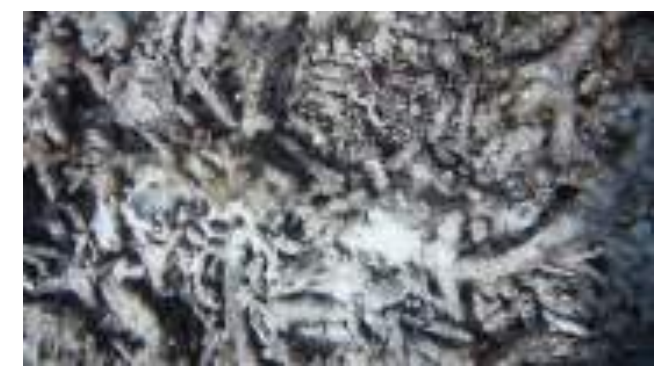

(b)

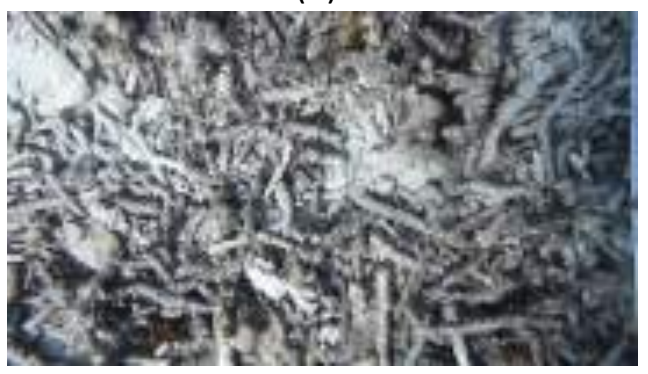

(d)

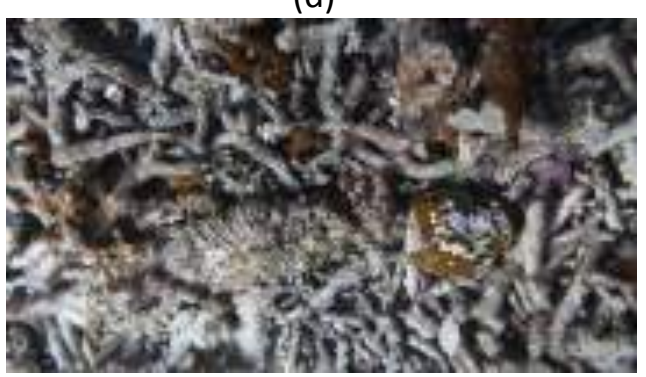

(f)

Figure 1(a), (b), (c), (d), (e) and (f) image of rubble in coral reef from INOS

\section{Edge Detection}

Edges are the main parts of an image which show variations in the objects. Physical edges are produced by variation in the reflectance, orientation, illumination, and depth of scene surfaces. There are various edge detection techniques. A study by Hussain and Agarwal (2015) reviewed different edge detection techniques. They concluded that the Sobel,
Robert and Prewitt were able to detect edges along their orientation, but the results were not accurate and they were sensitive to noise. Canny edge detection however is more complex compared to Sobel, Robert and Prewitt methods but has the slight advantages in term of noise immunity. After the preliminary experiment, Prewitt edge detection could provide better results compared to others so it was employed in this study. Figure 2(a), 
(b), (c) and (d) show the original images and the results after Sobel, Robert and Prewitt techniques, respectively.

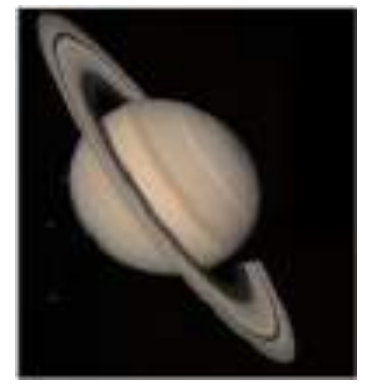

(a)

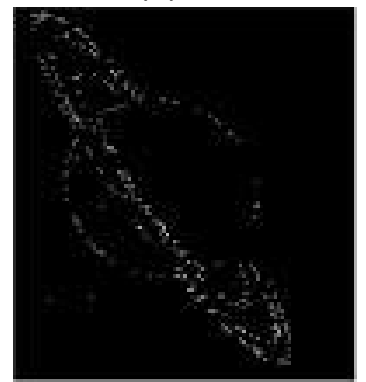

(c)

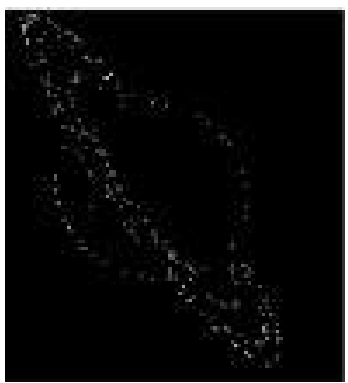

(b)

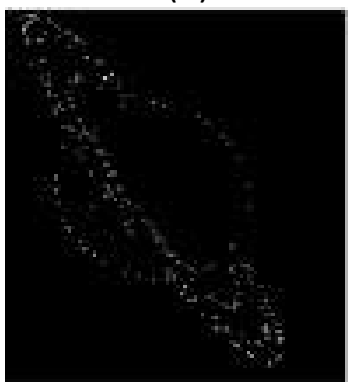

(d)

Figure 2: (a) The Original Image, (b) Resulting Image after Sobel Technique, (c) Resulting Image after Robert Technique and (d) Resulting Image after Prewitt Technique

\section{Morphological Dilation and Erosion}

Morphological operations apply a dedicated component called a structuring component to an input image to either process a target object with bigger or smaller size depending on the shape and size of the structuring element. The shape and size of these structuring elements must be carefully selected so that it yields a desired result. Thus, by selecting and arranging the correct size and shape of the structuring element for a targeted area to be processed in the image, a set of morphological operation can be produced to process certain target shape in the image.

In this paper, two basic morphological operation were used, namely dilation and erosion. Dilation basically expands the targeted object by adding pixels to the object.
On the other hand, erosion shrinks the object size by deleting the pixel from the target object. The expanded or shrunk targeted object size is dependent on the size and shape of the selected structuring element.

\section{Results and Discussion \\ Experimental Results}

The experimental results of this study were obtained using MATLAB software. Figure 4.1 shows the original image of rubble in a coral reef environment which was captured in Chagar Hutang, Redang Island, Terengganu. In the original image, a tape shown in the red circle that was used for Coral Video Transect was included. The light blue circles showed the shadow effects created due to the overlapping of corals. The shadow effects would affect the image complement process. 


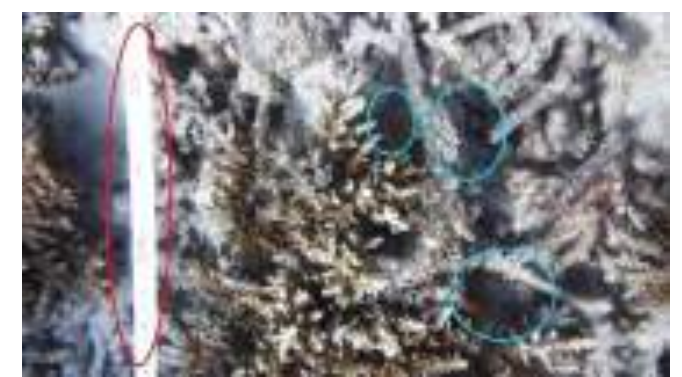

Figure 3: Original image of coral reef in Chagar Hutang, Redang island, Terengganu

Figure 4 shows the complement image of Figure 3. As expected, the shadow effects affected the process of image complement because the shadow became a dull territory in the image, causing potential rubble to be hidden. However, sand area was a lighter region that needed to be removed. Figure 4 shows that the sand area has been converted from light region to a dark region. This phenomenon would aid in image segmentation.

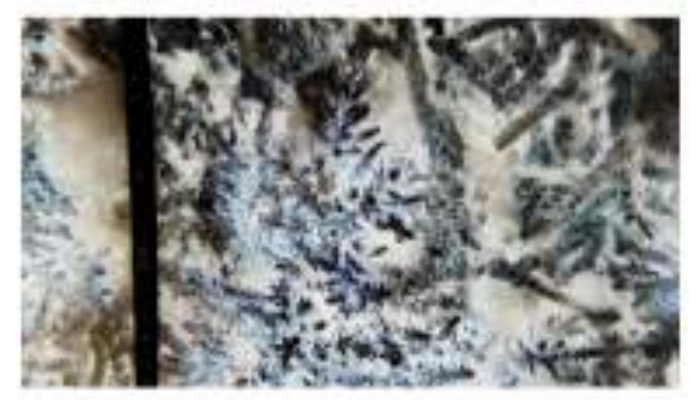

Figure 4: Complement image of Figure 3

Figure 5 shows the result of Figure 4 after undergoing the process of thresholding. Thresholding helps to clarify the colour of regions by converting the image to black and white. In other words, the RGB image is converted into a binary image. In this case, colour was not considered because coral reef rubble could be any colour but less than $5 \mathrm{~cm}$ in length. The tape that was shown in the red circle in Figure 3 was completely black. This would ease in image segmentation process.

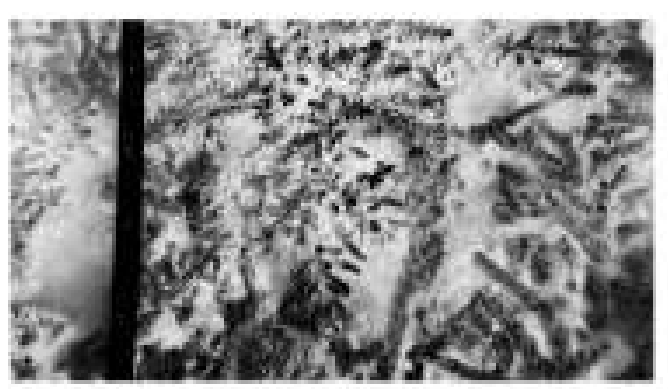

Figure 5 Binary Image of Figure 4

Figure 6 shows the result of Figure 5 after undergoing the process of image segmentation with the use of edge detection. As mentioned before, Prewitt edge detector is the most suitable. Prewitt edge detector has shown the most edges in the image. However, the edges found could contain image noises and the tape outer line shown in the red circle in Figure 6 was still visible. Other than that, the lighter regions in Figure 5 had been completely removed in Figure 6. 


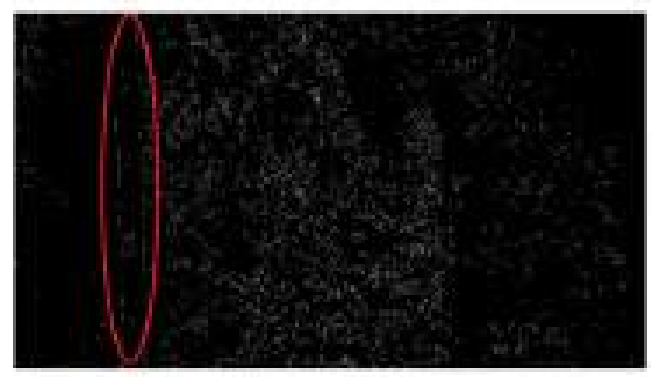

Figure 6: Black and white image of Figure 4 after applying Prewitt edge detector

Figure 7 shows the result of Figure 6 after undergoing the process of noise smoothing. Wiener filter was used to remove the noise and deblur the image simultaneously. The white pixels in Figure 7 appeared larger compared to
Figure 6. With the noise removed, the gap between white pixels and black pixels was clearly visible instead of the white dots that resulted in Figure 6.

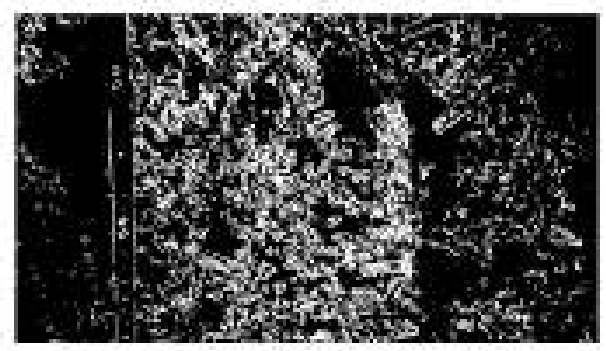

Figure 7: Image of Figure 6 after applying wiener filter

Figure 8 shows the result of Figure 7 after undergoing the process of erosion. In Figure 8, the white pixels had been reduced by the use of structural element that was set to a pre-defined measurement for rubbles. However, the predefined measurement was slightly inaccurate due to lack of knowledge. Therefore, potential rubbles could be left out.

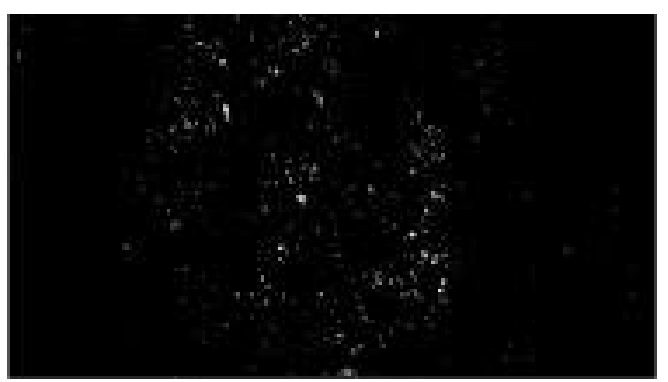

Figure 8: Image of Figure 7 after erosion

Figure 9 shows the result of Figure 8 after undergoing the process of dilation. Due to the pre-defined measurement of the white pixels, dilation process added pixels in the pixel's neighborhood to a state whereby the white pixels could be visibly seen as a consistent form. 


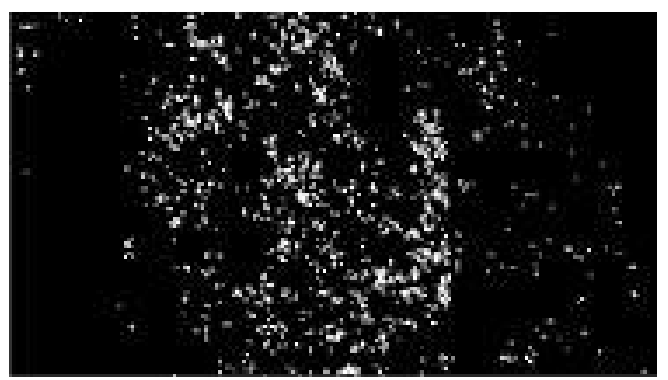

Figure 9: Image of Figure 8 after dilation

Figure 10 shows the sample interface done using GUI in MATLAB and the average percentage of rubble coverage. The average percentage of rubble coverage was obtained by counting the total of white pixels in Figure 9 over the total pixels of Figure 9. The sample has a few buttons for the user to select image and process image. The "select image" button will open a file of sample original images from
Chagar Hutang and the original image is shown in the original image axes. Next, the user can press the "process image" button to process the image and the processed image is shown in the processed image axes. By pressing the "process image" button, the result for average percentage of rubble coverage is automatically counted and shown. The interface is simple and easy to use for experts and non-experts.

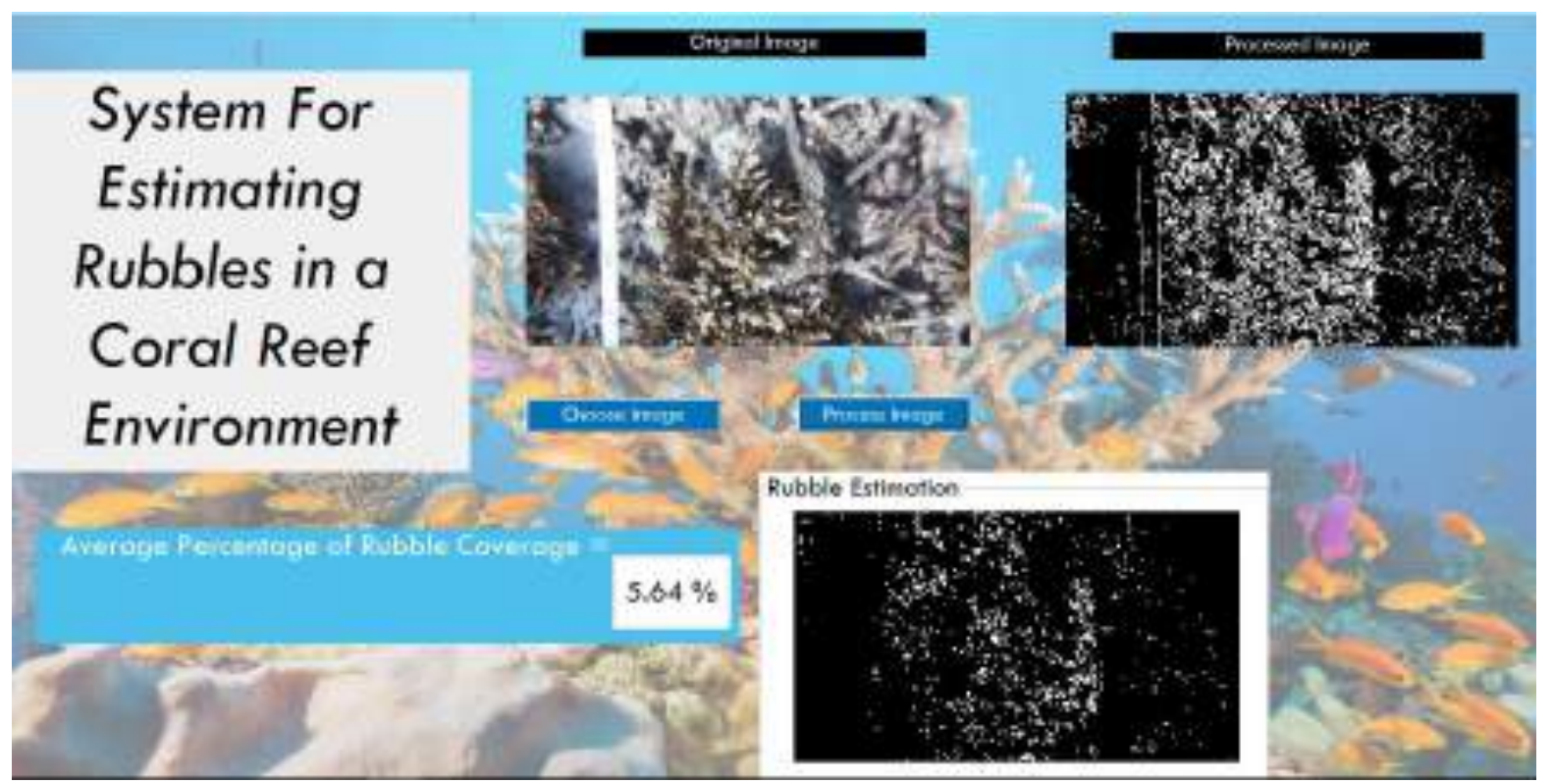

Figure 10: GUI of the Prototype System

The following figure shows other samples of image that had been tested with the same methods. Figure 11(a), (b), (c) and (d) show the original images of rubble in a coral reef environment that was also taken in Chagar Hutang, Redang Island, Terengganu. The light blue circles showed that the image had shadow effects similar to Figure 3. Moreover, a few blur effects were shown as well. This might cause the estimation of rubble to be slightly inaccurate. 


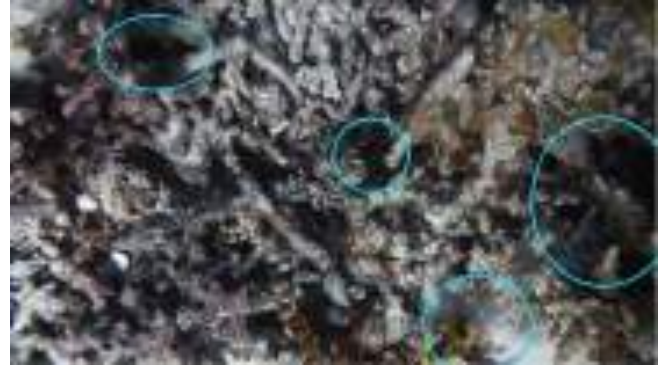

(a)

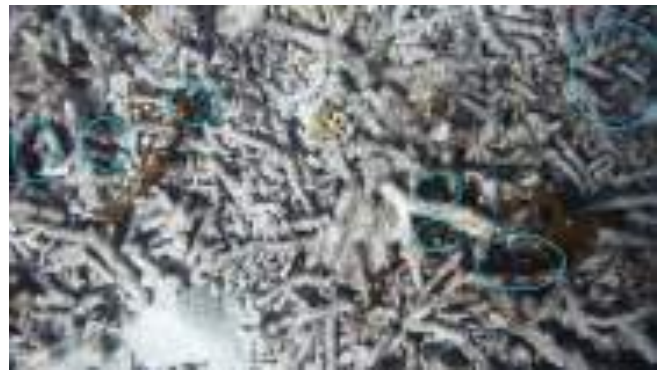

(c)

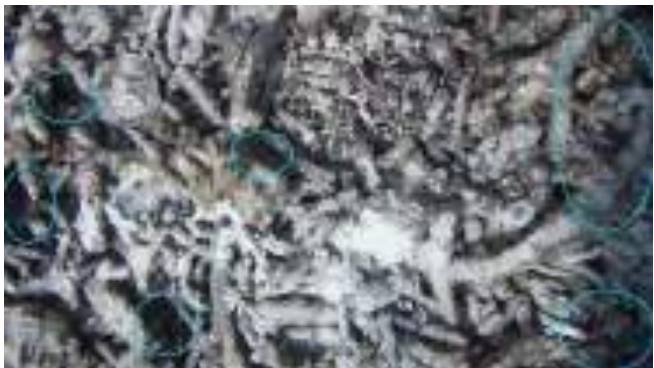

(b)

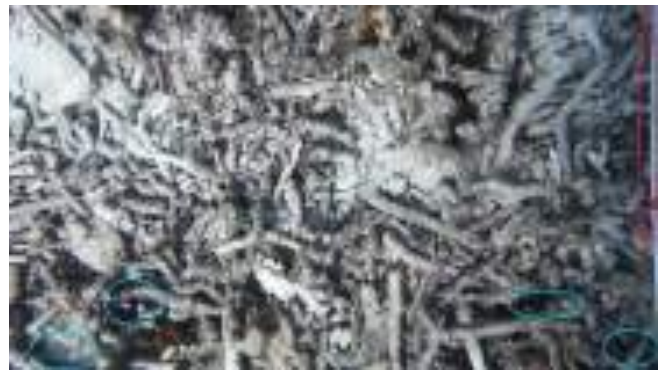

(d)

Figure 11: (a), (b), (c) and (d) Original images of coral reef in Chagar Hutang, Redang Island

Figure 12(a), (b), (c) and (d) show the complement images of Figure 11(a), (b), (c) and (d), respectively. The shadow effects affected these images as well. The blur effect slightly affected the image complement process but could be solved by noise smoothing. The

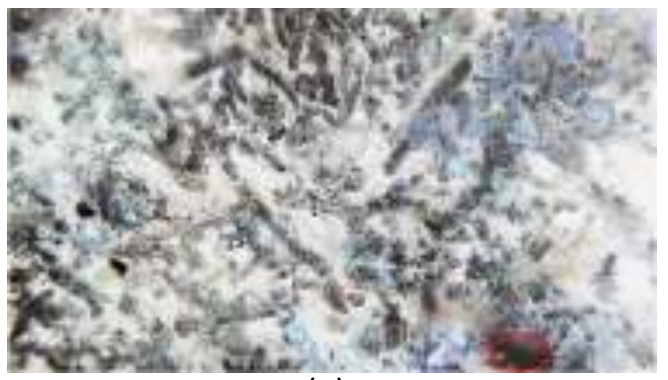

(a)

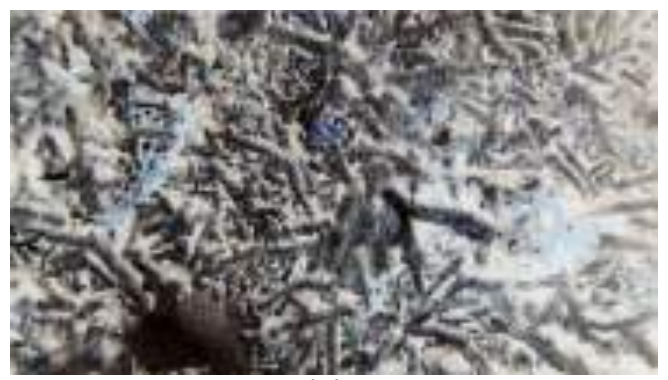

(c) red circle shown in Figure 12 (a) was the sand area. The sand area clearly converted from a lighter region to a dark region. This shows that the removal of sand was successful but still needed improvement.

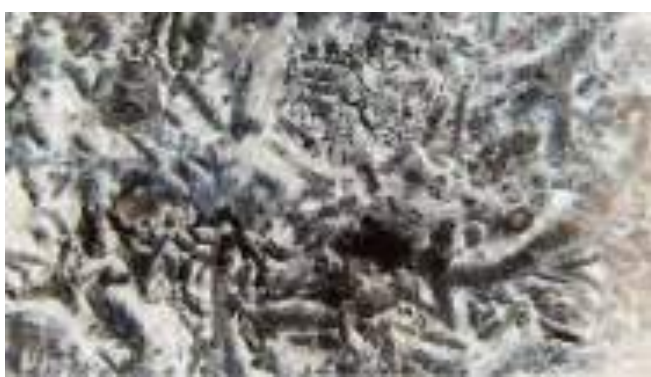

(b)

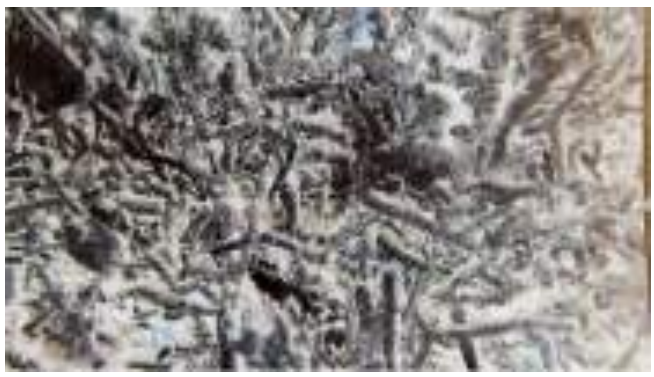

(d)

Figure 12: (a) Complement image of Figure 11 (a), (b) Complement image of Figure 11(b), (c) Complement image of Figure 11(c) and (d) Complement image of Figure 11(d) similar as shown in Figure 5. The sand area had

Figure 13(a), (b), (c) and (d) show the results of Figure 12(a), (b), (c) and (d) after undergoing the process of thresholding, respectively. The results had been proven to be become dark regions. Therefore, the removal of sand was still successful for these following images. 
Figure 14 (a), (b), (c) and (d) show the results of Figure 13(a), (b), (c) and (d) after undergoing the process of image segmentation which was edge detection, respectively. The edges were clearly shown in Figure 14(a), (b), (c) and (d). However, the edges found could still be image noises. Figure 14(d) consists of unwanted object such as the coral video transect tape shown in red circle of Figure 11(d).

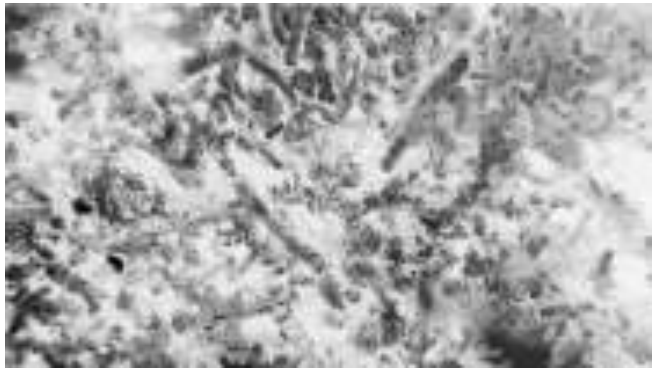

(a)

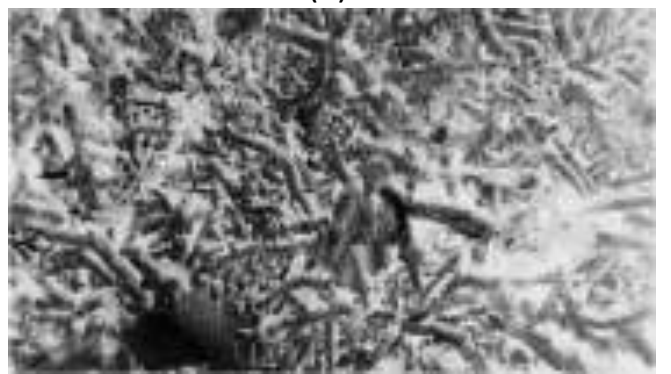

(c)
Figure 15(a), (b), (c) and (d) show the results of Figure 15(a), (b), (c) and (d) after applying a noise smoothing technique which was Wiener filtering, respectively. The gaps between black and white pixels could clearly be seen as well. However, the coral video transect tape was still visible in Figure 15(d). Wiener filter was not suitable for Figure 15(d)

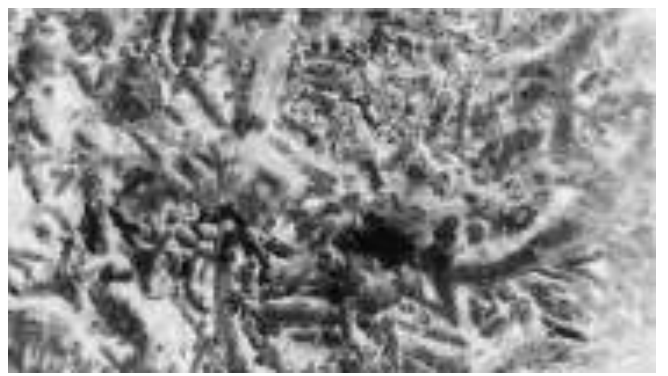

(b)

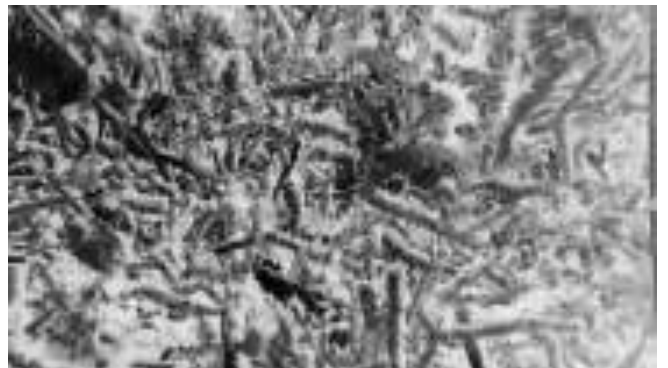

(d)

Figure 13: (a) Binary image of Figure 12(a), (b) Binary image of Figure 12(b), (c) Binary image of Figure 12(c) and (d) Binary image of Figure 12(d)

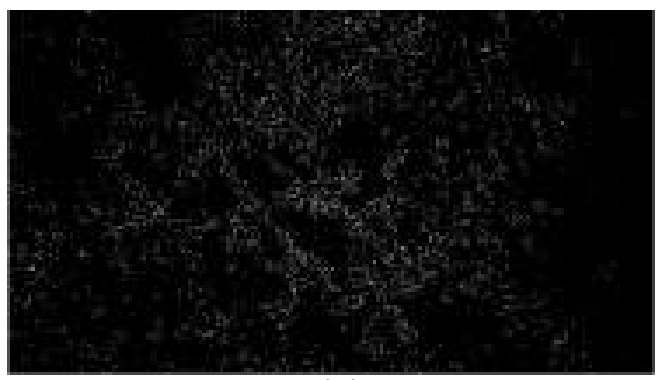

(a)

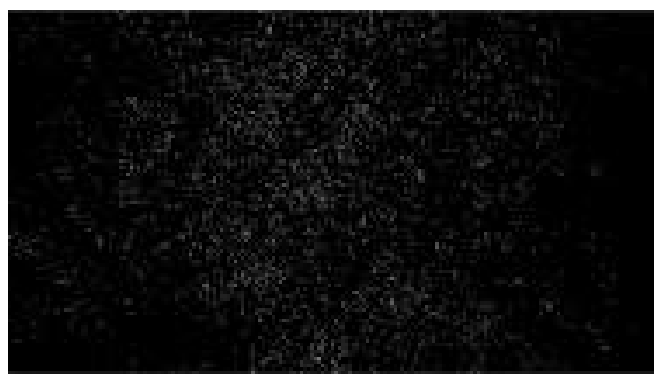

(c)

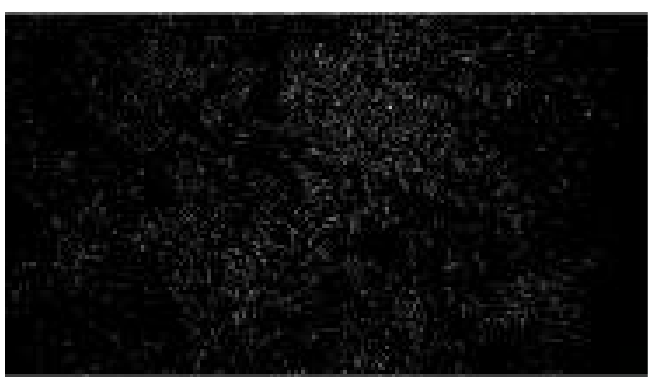

(b)

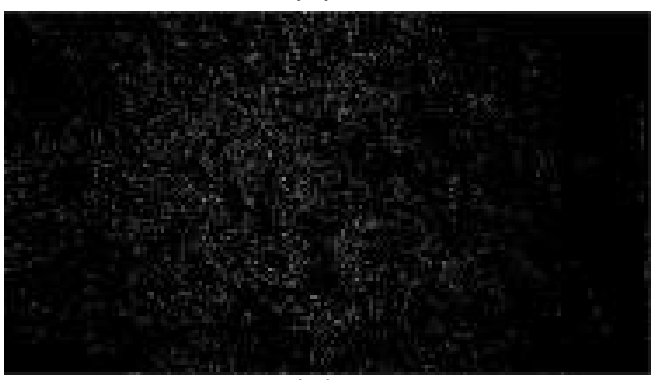

(d)

Figure 14: (a) Black and white image of Figure 13(a) after applying Prewitt edge detector, (b) Black and white image of Figure 13(b) after applying Prewitt edge detector, (c) Black and white image of Figure 13(c) after applying Prewitt edge detector and (d) Black and white image of Figure 13(d) after applying Prewitt edge detector 


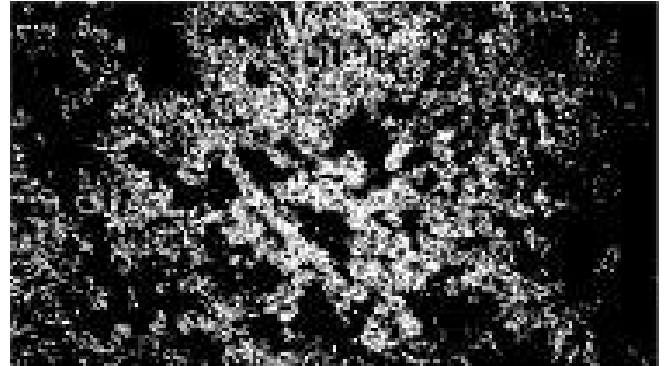

(a)

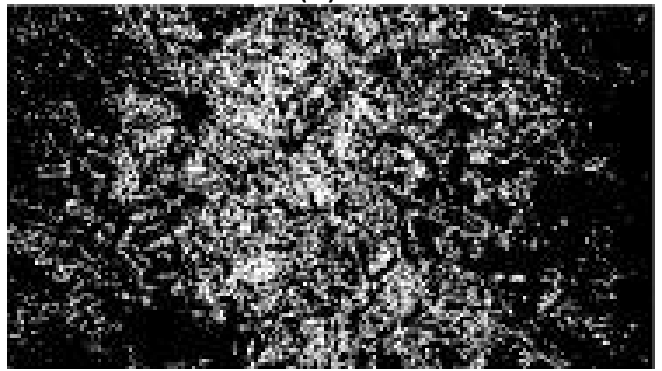

(c)

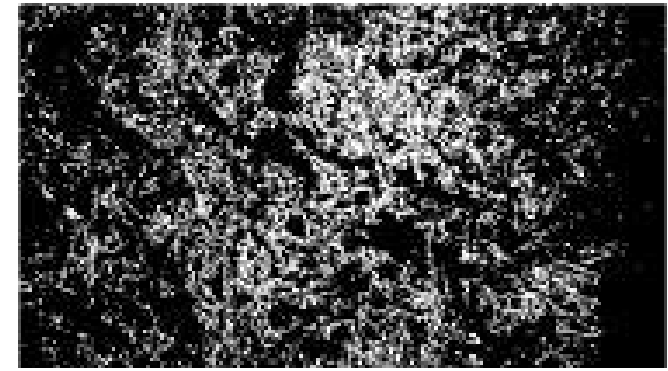

(b)

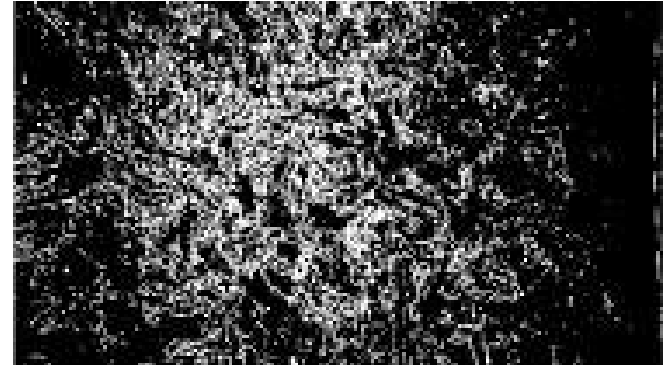

(d)

Figure 15: (a) Image of Figure 14(a) after applying Wiener filter, (b) Image of Figure 14(b) after applying Wiener filter, (c) Image of Figure 14(c) after applying Wiener filter and (d) Image of Figure 14(d) after applying Wiener filter

Figure 16(a), (b), (c) and (d) show the results of Figure 14(a), (b), (c) and (d) after applying erosion method, respectively. For Figure 16(a), (b), (c), the result had been proven to be similar to Figure 8. In this case, erosion method clearly worked for Figure 16(d) for removing the coral video transect tape shown in the red circle in Figure 11(d). There were no existing white pixels detected at the tape area. Therefore, the erosion method showed potentially excellent results. However, the white pixels shown in Figure 16(a), (b), (c) and (d) were unorganized in the form of rubble. Dilation method was needed to enhance the white pixels so that it could be clearly visible or detected.

Figure 17(a), (b), (c) and (d) show the results of Figure 16(a), (b), (c) after applying dilation method, respectively. The white pixels could be seen clearly and the rubble coverage could be counted from these results. 


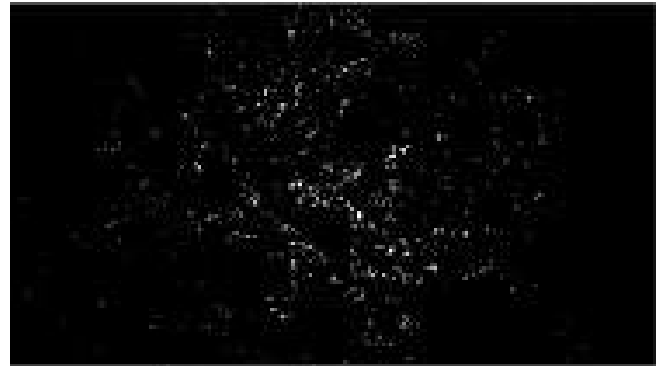

(a)

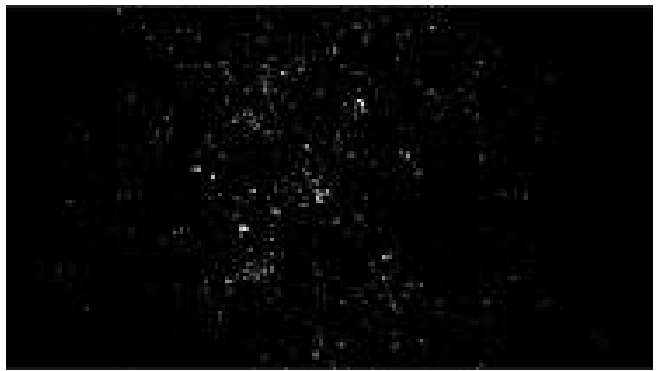

(c)

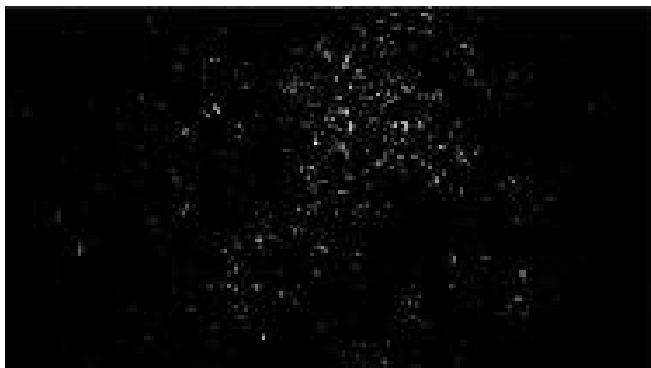

(b)

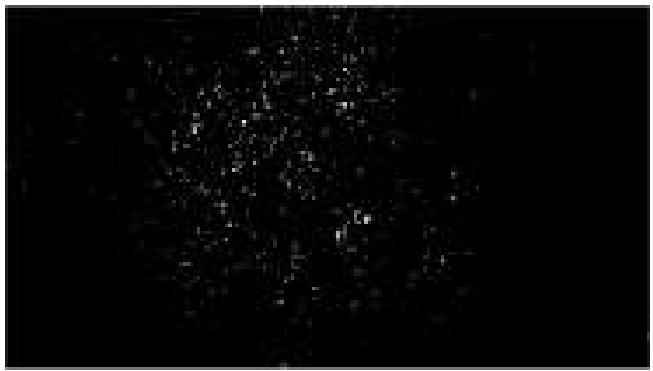

(d)

Figure 16: (a) Image of Figure 15(a) after erosion, (b) Image of Figure 15(b) after erosion, (c) Image of Figure 15(c) after erosion and (d) Image of Figure 15(d) after erosion

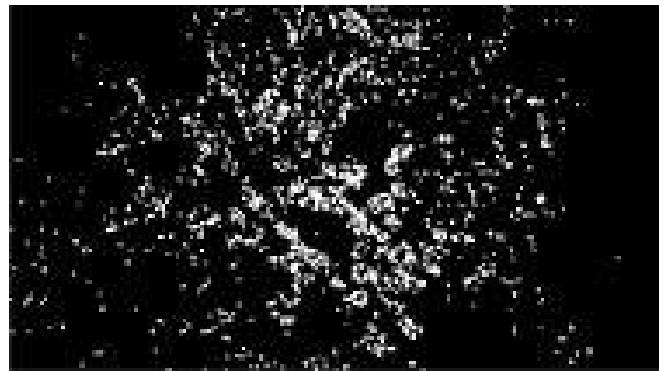

(a)

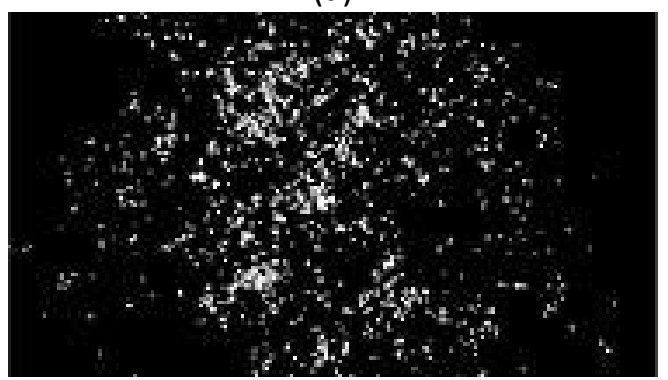

(c)

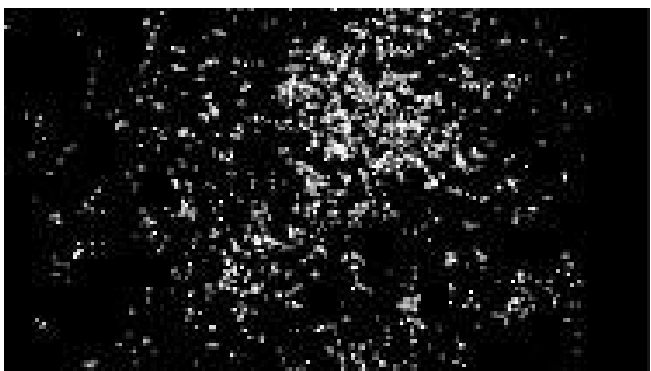

(b)

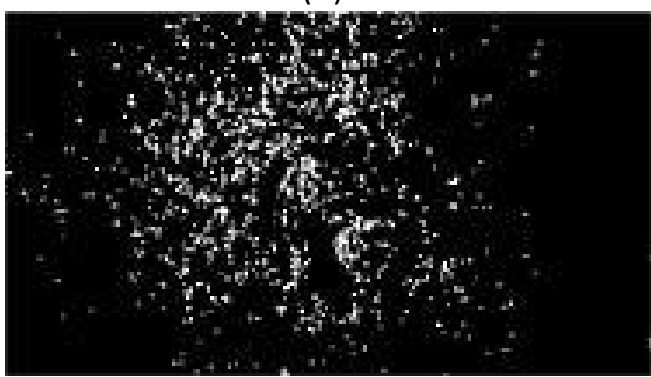

(d)

Figure 17: (a) Image of Figure 16(a) after dilation, (b) Image of Figure 14(b) after dilation, (c) Image of Figure 16(c) after dilation, and (d) Image of Figure 16(d) after dilation

\section{Discussion}

A common conventional method used to estimate reef rubble coverage is by using Coral Point Count with Excel extension (CPCe). However, this conventional method takes long hours to obtain the estimated results. Other than that, expertise is required to use the specific software. Hence, this paper presents a new alternative way of estimating the quantity of reef rubble coverage from still images. This study found that the average result obtained by INOS was $8.16 \%$ of rubble coverage area in the still images and the average result obtained by the proposed method in this paper is $5.74 \%$. The results are based on the average percentage of rubble coverage in coral reef images after 20 images of coral reef were tested using the proposed method. It should be noted that there are still some flaws in the proposed methods. 
Some parts of the image that was reef rubble but undetected by the proposed method and some parts might be dead corals but identified as the white pixels. This result was due to the process of binarization and object detection that was used in this work being still very crude; thus, some information was still missing and the method needed further improvement in the future. The proposed method was based on edge detection and morphology including complementing, thresholding, edge detection, wiener filtering, erosion and dilation. However, these methods were still not enough to accurately estimate the reef rubble coverage in the still images.

Among the challenges faced in detecting reef rubble were illumination differences in an underwater environment, variation in image quality due to floating particles in underwater environment and reef rubble existing in various forms. Therefore, more specific techniques were needed to process the image. For example, image taken at different depth of underwater environment would lead to different colour due to light absorption. Thus, a specific technique that could filter out or provide the light absorption recalculation may be needed to enhance the image quality so that reef rubbles could be detected.

\section{Conclusion}

In this paper, a system for estimating the rubble coverage in a still image was successfully developed using MATLAB software. This system included 6 steps which were image complement, thresholding, edge detection, wiener filtering, erosion and dilation. The first phase of the method was complementing the image. Image complement process is useful to remove sand from the image. However, if there are shadows in the image, the system may not be able to accurately remove the noise due to lack of brightness in the image. The second phase was thresholding that converted the RGB image to black and white. Therefore, some portion of information would be missing in this process due to the nature of binary thresholding process. The third phase was edge detection.
There are many edge detectors, but in this work only Prewitt, Sobel and Roberts were tested. Prewitt edge detectors showed more convincing results than the others. Therefore, Prewitt was chosen. The edge detectors might have detected noise as well. To remove the noise, Wiener filter was applied to the image. Wiener filter removes noise in the image, indirectly causing the pixels to be larger. After noise was removed, there were still many particles presented that were not part of rubble. So, by introducing erosion and dilation morphological operation, it was hoped that only the actual rubble would be detected.

Erosion and dilation use structuring element to an input image, creating an output image of the same size. Dilation adds pixels to the boundaries of objects in an image, while erosion removes pixels on object boundaries. The number of pixels added or removed from the objects in an image depends on the size and shape of the structuring element used to process the image. The final step was calculating the rubbles by dividing the total pixels in the image over the white pixels in the image.

Based on the outcome obtained, we can assume that the average results were slightly different than the results produced by experts from INOS. The average result obtained by INOS was $8.16 \%$ while the average result obtained by the proposed method was $5.74 \%$. The results showed some differences since the proposed method was a semi-automatic approach that lost some information in the process due to the complexity of the rubble image. It should be noted that the manual approach is also not precisely accurate since it is just an estimated approach. Overall, this work has shown that image processing could be used to estimate coral reef rubble coverage that could reduce time consumption in manual surveys, lower cost as well and used by nonexperts. However, further research and development is still needed in the future to further improve the accuracy of the estimation. 


\section{Acknowledgements}

We would like to thank the Institute of Oceanography and Environment (INOS),

\section{References}

Alcala, A. C., \& Gomez, E. D. 1982. Coral growth-rates on a reef flat in Sumilon Island, Central Philippines. Kalikasan-The Philippine Journal of Biology, 11(1), 179184.

Benediktsson, J. A., Pesaresi, M., \& Amason, K. 2003. Classification and feature extraction for remote sensing images from urban areas based on morphological transformations. IEEE Transactions on Geoscience and Remote Sensing, 41(9), 1940-1949.

Bierwirth, P. N., Lee, T. J., \& Burne, R. V. 1993. Shallow sea-floor reflectance and water depth derived by unmixing multispectral imagery. Photogrammetric Engineering and Remote Sensing ;( United States), 59(3).

Carleton, J. H., \& Done, T. J. 1995. Quantitative video sampling of coral reef benthos: large-scale application. Coral Reefs, 14(1), 35-46.

Carper, W., Lillesand, T., \& Kiefer, R. 1990. The use of intensity-hue-saturation transformations for merging SPOT panchromatic and multispectral image data. Photogrammetric Engineering and remote sensing, 56(4), 459-467.

Chavez, P., Sides, S. C., \& Anderson, J. A. 1991. Comparison of three different methods to merge multiresolution and multispectral data- Landsat TM and SPOT panchromatic. Photogrammetric Engineering and remote sensing, 57(3), 295303.

Green, E. P., Mumby, P. J., Edwards, A. J., \& Clark, C. D. 1996. A review of remote sensing for the assessment and management
Universiti Malaysia Terengganu (UMT) for providing the data and information on coral reef images.

of tropical coastal resources. Coastal management, 24(1), 1-40.

Haralick, R. M. 1979. Statistical and structural approaches to texture. Proceedings of the IEEE, 67(5), 786-804.

Hussain, Z., \& Agarwal, D. 2015. A comparative analysis of edge detection techniques used in flame image processing. International Journal of Advance Research in Science and Engineering IJARSE, (4).

Kohler, K. E., \& Gill, S. M. 2006. Coral Point Count with Excel extensions (CPCe): A Visual basic program for the determination of coral and substrate coverage using random point count methodology. Computers \& Geosciences, 32(9), 1259-1269.

Lenth, R. V. 2001. Some practical guidelines for effective sample size determination. The American Statistician, 55(3), 187-193.

Mausel, P. W., Kramber, W. J., \& Lee, J. K. 1990. Optimum band selection for supervised classification of multispectral data. Photogrammetric Engineering and Remote Sensing, 56, 55-60.

Mumby, P. J., \& Harborne, A. R. 1999. Development of a systematic classification scheme of marine habitats to facilitate regional management and mapping of Caribbean coral reefs. Biological conservatio, 88(2), 155-163.

Rasser, M., \& Riegl, B. 2002. Holocene coral reef rubble and its binding agents. Coral Reefs, 21(1), 57-72.

Soriano, M., Marcos, S., Saloma, C., Quibilan, M., \& Alino, P. 2001. Image classification 
of coral reef components from underwater color video. In Oceans, 2001. MTS/IEEE Conference and Exhibition (Vol. 2, pp. 1008-1013). IEEE.

MathWork. 1994 - 2018. Morphological Dilation and Erosion. https://www.mathworks.com/help/images/ morphological-dilation-and-erosion.html [25 April 2018]
Vogt, P., Riitters, K. H., Estreguil, C., Kozak, J., Wade, T. G., \& Wickham, J. D. 2007. Mapping spatial patterns with morphological image processing. Landscape ecology, 22(2), 171-177.

Woodley, J., Chornesky, E., Cliffo, P., Jackson, J., Kaufman, L., Knowlton, N., \& Rylaarsdz, K. W. 1981. Hurricane Allen's impact on a Jamaican coral reef. Science, 214, 13. 